\title{
On Some Fixed Point Theorems for 1-Set Weakly Contractive Multi-Valued Mappings with Weakly Sequentially Closed Graph
}

\author{
Afif Ben Amar ${ }^{1}$, Aneta Sikorska-Nowak ${ }^{2}$ \\ ${ }^{1}$ Département de Mathématiques Université de Gafsa Faculté des sciences de Gafsa Cité \\ Universitaire Zarrouk, Gafsa Tunisie \\ ${ }^{2}$ Faculty of Mathematics and Computer Science, Adam Mickiewicz University, Poznan, Poland \\ E-mail: afif.benamar@ipeis.rnu.tn, anetas@amu.edu.pl \\ Received February 20, 2011; revised March 11, 2011; accepted March 21, 2011
}

\begin{abstract}
In this paper we prove Leray-Schauder and Furi-Pera types fixed point theorems for a class of multi-valued mappings with weakly sequentially closed graph. Our results improve and extend previous results for weakly sequentially closed maps and are very important in applications, mainly for the investigating of boundary value problems on noncompact intervals.
\end{abstract}

Keywords: Measures of Weak Noncompactness, Weakly Condensing, Weakly Nonexpansive

\section{Introduction}

Fixed point theory for weakly completely continuous multi-valued mappings takes an important role for the existence of solutions for operator inclusions, positive solutions of elliptic equation with discontinuous nonlinearities and periodic and boundary value problems for second order differential inclusions (see [1-3]) and others. In [4] O’Regan has proved a number of fixed point theorems for multi-valued maps defined on bounded domains with weakly compact convex values and which are weakly contractive and have weakly sequentially closed graph. The aim of the present paper is to extend and improve these theorems to the case of weakly condensing and 1-set weakly contractive multi-valued maps with weakly sequentially closed graph. Furthermore, we do not assume that they are from a point into weakly compact convex set. We note that the domains of all of the multi-valued maps discussed here are not assumed to be bounded. Our results generalize and extend relevant and recent ones (see [4-10]). The main condition in our results is formulated in terms of axiomatic measures of weak noncom- pactness.

Now we shall introduce the notation and give preliminary results which will be needed in the paper.

Let $X$ be a Hausdorff linear topological space, then we define

$$
\begin{aligned}
& P_{\mathrm{bd}}(X)=\{A \subset X: A \text { is non-empty, and bounded }\} \\
& P_{\mathrm{cv}}(X)=\{A \subset X: A \text { is non-empty, and convex }\} \\
& P_{\mathrm{cl}, \mathrm{cv}}(X) \\
& =\{A \subset X: A \text { is non-empty, closed, and convex }\}
\end{aligned}
$$

Let $Z$ a non-empty subset of a Banach space $Y$ and $F: Z \rightarrow 2^{X}$ be a multi-valued mapping. We denote

$$
R(F)=\bigcup_{y \in Z} F(y) \text { and } \operatorname{Gr} F=\{(z, x) \in Z \times X: x \in F(z)\}
$$

the range and the graph of $F$ respectively. Moreover, for every subset $A$ of $X$, we put

$F^{-1}(A)=\{z \in Z: F(z) \cap A \neq \varnothing\}$ and

$F^{+}(A)=\{z \in Z: F(z) \subset A\}$.

- $F$ is called upper semicontinuous on $Z$ if $F^{-1}(A)$ is closed, for every closed subset $A$ of $X$ (or, equivalently, if $F^{+}(A)$ is open, for every open subset $A$ of $X)$.

- $F$ is called weakly upper semicontinuous if $F$ is upper semicontinuous with respect to the weak topologies of $Z$ and $X$.

Now we suppose that $X$ is a Banach space and $Z$ is weakly closed in $Y$.

$F$ is said to be weakly compact if the set $R(F)$ is relatively weakly compact in $X$. Moreover, $F$ is said 
to have weakly sequentially closed graph if for every sequence $\left(x_{n}\right)_{n} \subset Z$ with $x_{n} \stackrel{w}{\longrightarrow} x$ in $Z$ and for every sequence $\left(y_{n}\right)_{n}$ with

$y_{n} \in F\left(x_{n}\right), \forall n \in \mathbb{N}, y_{n} \stackrel{w}{\longrightarrow} y$ in $X$ implies $y \in F(x)$, where $\stackrel{w}{\longrightarrow}$ denotes weak convergence. $F$ is called weakly completely continuous if $F$ has a weakly sequentially closed graph and, if $A$ is bounded subset of $Z$, then $F(A)$ is a weakly relative compact subset of $X$.

Definition 1.1 Let $E$ be a Banach space and $C$ a lattice with a least element, which is denoted by 0 . By a measure of weak non-compactness (MNWC) on E, we mean a function $\Phi$ defined on the set of all bounded subsets of $E$ with values in $C$, such that for any $\Omega_{1}, \Omega_{2} \in P_{\mathrm{bd}}(E)$ :

(1) $\Phi\left(\overline{\operatorname{conv}}\left(\Omega_{1}\right)\right)=\Phi\left(\Omega_{1}\right)$, where $\overline{\operatorname{conv}}$ denotes the closed convex hull of $\Omega$.

(2) $\Omega_{1} \subseteq \Omega_{2} \Rightarrow \Phi\left(\Omega_{1}\right) \leq \Phi\left(\Omega_{2}\right)$,

(3) $\Phi\left(\Omega_{1} \cup\{a\}\right)=\Phi\left(\Omega_{1}\right)$ for all $a \in E$.

(4) $\Phi\left(\Omega_{1}\right)=0$ if and only if $\Omega_{1}$ is weakly relatively compact in $E$.

If the lattice $C$ is a cone of vector space, then the MNWC $\Phi$ is said positive homogenous provided $\Phi(\lambda \Omega)=\lambda \Phi(\Omega)$ for all $\lambda>0$ and $\Omega \in P_{\text {bd }}(E)$.

The notion above is a generalization of the important well known DeBlasi measure of weak non-compactness $\beta$ (see [11]) defined on each bounded set $\Omega$ of $E$ by

$$
\begin{aligned}
\beta(\Omega)= & \inf \{\varepsilon>0: \text { there exist sa weakly } \\
& \text { compact set } \left.D \text { such that } \Omega \subset D+\varepsilon B_{E}\right\}
\end{aligned}
$$

where $B_{E}$ is the closed unit ball of $E$.

It is well known that $\beta$ enjoys these properties: for any $\Omega_{1}, \Omega_{2} \in P_{\text {bd }}(E)$,

(5). $\beta\left(\Omega_{1} \cup \Omega_{2}\right)=\max \left\{\beta\left(\Omega_{1}\right), \beta\left(\Omega_{2}\right)\right\}$.

(6) $\beta\left(\lambda \Omega_{1}\right)=\lambda \beta\left(\Omega_{1}\right)$ for all $\lambda>0$.

(7) $\beta\left(\Omega_{1}+\Omega_{2}\right) \leq \beta\left(\Omega_{1}\right)+\beta\left(\Omega_{2}\right)$.

Now let us introduce the following definitions.

Definition 1.2 Let $\Omega$ be a nonempty subset of Banach space $E$ and $\Phi$ a MNWC on E. If $F: \Omega \rightarrow 2^{E}$, we say that

(a) $F$ is $\Phi$-condensing if $\Phi(F(D))<\Phi(D)$ for all bounded sets $D \subseteq \Omega$ with $\Phi(D) \neq 0$.

(b) $F$ is $\Phi$-nonexpansive map if $\Phi(F(D)) \leq \Phi(D)$ for all bounded sets $D \subseteq \Omega$.

In the sequel, we shall need also the following multivalued fixed point theorem.

Theorem 1.1 (see [12], p. 206). Let $\Omega$ be a nonvoid, convex and closed subset of a locally convex space $E$. Let $F: \Omega \rightarrow P_{\mathrm{cl}, \mathrm{cv}}(\Omega)$ be an upper semicontinuous multi-valued mapping such that $F(\Omega)$ is relatively compact. Then $F$ has a fixed point.

\section{Sadovskii Type Fixed Point Theorems}

We begin with the following interesting property of multi-valued maps with weakly sequentially closed graph which is a basic tool for achieving our aim.

Theorem 2.1 Let $\Omega$ be a non-empty, weakly compact subset of a Banach space E. Suppose $F: \Omega \rightarrow 2^{E}$ has weakly sequentially closed graph and $F(\Omega)$ is relatively weakly compact. Then $F$ has a weakly closed graph.

Proof. Since $(E \times E)_{w}=E_{w} \times E_{w}$ ( $E_{w}$ the space $E$ endowed its weak topology), it follows that $\Omega \times F(\Omega)^{w}$ is a weakly compact subset of $E \times E$. Also,

$G r F \subset \Omega \times \overline{F(\Omega)^{w}}$. So, $G r F$ is relatively weakly compact. Let $(x, y) \in \Omega \times \overline{F(\Omega)^{w}}$ be weakly adherent to $G r F$, then by the Eberlein-Šmulian theorem [13, theorem

8.12.4, p. 549] we can find $\left\{\left(x_{n}\right),\left(y_{n}\right)\right\}_{n} \subseteq G r F$ such that $y_{n} \in F\left(x_{n}\right), x_{n} \stackrel{w}{\longrightarrow} x$ and $y_{n} \stackrel{w}{\longrightarrow} y$ in $E$. Because $F$ has weakly sequentially closed graph, $y \in F(x)$ and so $(x, y) \in G r F$. Therefore, $G r F$ is weakly closed.

Q.E.D.

Remark 2.1 With the conditions of Theorem 2.1, we prove that $\mathrm{GrF}$ is weakly compact.

Theorem 2.2 Let $\Omega$ be a non-empty, closed, convex subset of a Banach space $E$. Suppose $F: \Omega \rightarrow P_{\mathrm{cv}}(\Omega)$ has weakly sequentially closed graph and $F(\Omega)$ is weakly relatively compact. Then $F$ has a fixed point.

Proof. Set $K=\overline{\operatorname{conv}}(F(\Omega))$. It follows, using the Krein-Šmulian theorem (see [14, p. 434]) that $K$ is a weakly compact convex set. We have $F(\Omega) \subseteq K \subseteq \Omega$. Notice also that $F: K \rightarrow P_{\mathrm{cv}}(K)$. By Theorem $2.1 F$ has weakly closed graph, and so $F(x)$ is weakly closed for every $x \in K$. Thus, by [15, Proposition 14.5, p. 69] $F$ is weakly upper semicontinuous. Because $E$ endowed with its weak topology is a Hausdorff locally convex space, we apply Theorem 1.1 to get that $F$ has a fixed point $x \in K \subseteq \Omega$.

Q.E.D.

Remark 2.2 (a) Theorem 2.2 improves Theorem 2.2 in [4] and Theorem 2.1 in [1] for the case of Banach spaces.

(b) Because every single-valued and weakly sequentially continuous map has weakly sequentially closed 
graph, then Theorem 2.2 extends and improves a fixed point theorem of Arino, Gautier and Penot for the case of Banach spaces [5], Theorem 2.5 in [9] and Corollary 2.3 in [10].

Now, we are ready to prove some fixed point theorems for a broader class of multi-valued mappings with weakly sequentially closed graph, in which the operators have the property that the image of any set is in a certain sense more weakly compact than the original set itself.

Theorem 2.3 Let $\Omega$ be a non-empty, closed, convex subset of a Banach space $E$. Assume $\Phi$ a MNWC on $E$ and $F: \Omega \rightarrow P_{\mathrm{cv}}(\Omega)$ has weakly sequentially closed graph. In addition, suppose that $F$ is $\Phi$-condensing and $F(\Omega)$ is bounded. Then $F$ has a fixed point.

Proof. Let $x_{0} \in \Omega$. We consider the family $F$ of all closed bounded convex subsets $D$ of $\Omega$ such that $x_{0} \in D$ and $F(x) \subseteq D$ for all $x \in D$. Obviously $F$ is non-empty, since $\overline{\operatorname{conv}}\left(F(\Omega) \cup\left\{x_{0}\right\}\right) \in F$. We denote $K=\underset{D \in F}{\cap} D$. We have that $K$ is closed convex and $x_{0} \in K$. If $x \in K$, then $F(x) \subseteq D$ for all $D \in F$ and hence $F(x) \subseteq K$. Consequently, $K \in F$. We will prove that $K$ is weakly compact. Denoting by

$K_{*}=\overline{\operatorname{conv}}\left(F(K) \cup\left\{x_{0}\right\}\right)$, we have $K_{*} \subseteq K$, which implies that $F(x) \subseteq F(K) \subseteq K_{*}$ for all $x \in K_{*}$. Therefore $K_{*} \in F$ and $K \subseteq K_{*}$. Consequently, $K=K_{*}$. Since $K$ is weakly closed, it suffices to show that $K$ is relatively weakly compact. If $\Phi(K)>0$, we obtain

$$
\Phi(K)=\Phi\left(\overline{\operatorname{conv}}\left(F(K) \cup\left\{x_{0}\right\}\right)\right) \leq \Phi(F(K))<\Phi(K)
$$

which is a contradiction. Hence, $\Phi(K)=0$ and so $K$ is relatively weakly compact. Now, $F: K \rightarrow P_{\mathrm{cv}}(K)$ has weakly sequentially closed graph. From Theorem 2.2, $F$ has a fixed point in $K \subseteq \Omega$.

Q.E.D.

Remark 2.3 Theorem 2.3 improves and extends Theorem 2.3 in [4].

Using Theorem 2.3, we obtain a sharpening of Theorem 2.2.

Corollary 2.1 Let $\Omega$ be a non-empty, closed, convex subset of a Banach space $E$. Assume $F: \Omega \rightarrow P_{\mathrm{cv}}(\Omega)$ a weakly completely continuous map with $F(\Omega)$ is bounded. Then $F$ has a fixed point.

Proof. This is an immediate consequence of Theorem 2.3, since $F$ is clearly $\Phi$-condensing where $\Phi$ is any MNWC on $E$.

\section{Q.E.D.}

Theorem 2.4 Let $E$ be a Banach space, $\Omega$ be a non-empty, closed, convex subset of $E$ and $\Phi$ a positive homogenous MNWC on E. Assume

$F: \Omega \rightarrow P_{\mathrm{cv}}(\Omega)$ has weakly sequentially closed graph, also suppose $F$ is $\Phi$-nonexpansive and $F(\Omega)$ is bounded. In addition, suppose that the implication

$$
\begin{aligned}
& f\left\{x_{n}\right\} \subset \Omega \text { with } y_{n} \in F\left(x_{n}\right) \text { for all } n \text { and } \\
& x_{n}-y_{n} \rightarrow \theta \text { as } n \rightarrow \infty,
\end{aligned}
$$

then there exists $x \in \Omega$ with $x \in F(x)$, holds. Then $F$ has a fixed point.

Remark 2.4 $\theta$ means the zero of the space $E$.

Proof. Let $F_{n}=t_{n} F$ for $n=1,2, \cdots$, where $\left(t_{n}\right)_{n}$ is a sequence of $(0,1)$ such that $t_{n} \rightarrow 1$. Since $\theta \in \Omega$ and $\Omega$ is convex, it follows that $\theta \in F_{n}: \Omega \rightarrow P_{\mathrm{cv}}(\Omega)$. Also $F_{n}$ has a weakly sequentially closed graph. Let $D \in P_{\text {bd }}(\Omega)$. Then, we have

$$
\Phi\left(F_{n}(D)\right)=\Phi\left(t_{n} F(D)\right) \leq t_{n} \Phi(D)
$$

So, if $\Phi(D) \neq 0$ we have

$$
\Phi\left(F_{n}(D)\right)<\Phi(D)
$$

Therefore, $F_{n}$ is $\Phi$-condensing on $\Omega$. From Theorem 2.3, $F_{n}$ has a fixed point, in $\Omega$. For all $n$, let $y_{n} \in F\left(x_{n}\right)$ with $x_{n}=t_{n} y_{n}$. Clearly the sequence $\left(y_{n}\right)_{n}$ is bounded and $x_{n}-y_{n}=\left(t_{n}-1\right) y_{n} \rightarrow \theta$ as $n \rightarrow \infty$, since $t_{n} \rightarrow 1$ as $n \rightarrow \infty$. Thus (1) implies that there exists $x \in \Omega$ with $x \in F(x)$.

Q.E.D.

As a consequence we obtain

Corollary 2.2 Let $\Omega$ be a non-empty, closed, convex subset of a Banach space $E$ and $\theta \in \Omega$. In addition assume $F: \Omega \rightarrow P_{\mathrm{cv}}(\Omega)$ has weakly sequentially closed graph, $\beta$-nonexpansive and $F(\Omega)$ is bounded. Also, suppose (1) holds. Then $F$ has a fixed point.

Proof. This is an immediate consequence of Theorem 2.4, since the DeBlasi measure $\beta$ is positive homogenous.

Q.E.D.

\section{Leray-Schauder and Furi-Pera Types Fixed Point Theorems}

In applications, the construction of a set $\Omega$ such that $F(\Omega) \subseteq \Omega$ is very difficult and sometimes impossible. In that line, we investigate maps $F: \Omega \rightarrow 2^{E}$ with weakly sequentially closed graph.

Lemma 3.1 Let $\Omega$ be a weakly closed subset of $a$ Banach space $E$ with $\theta \in \Omega$. Assume $F: \Omega \rightarrow 2^{E}$ has weakly sequentially closed graph with $F(\Omega)$ is bounded. Let $\left(x_{n}\right)_{n} \subseteq \Omega$ and $\left(\lambda_{n}\right)$ be a real sequence. If $x_{n} \stackrel{w}{\longrightarrow} x$ and $\lambda_{n} \rightarrow \lambda \in \mathbb{R}$, then the condition $x_{n} \in \lambda_{n} F\left(x_{n}\right)$ for all $n$ implies $x \in \lambda F(x)$.

Proof. For all $n$, there exists $y_{n} \in F\left(x_{n}\right)$ such that $x_{n}=\lambda_{n} y_{n}$. If $\lambda=0$, then $x_{n} \stackrel{w}{\longrightarrow} \theta \quad(F(\Omega)$ is bounded) and $x \in\{\theta\} \subseteq \Omega$. If $\lambda \neq 0$, then without loss 
of generality, we can suppose that $\lambda_{n} \neq 0$ for all $n$. So, $\lambda_{n}^{-1} x_{n}=y_{n}$ for all $n$ implies $y_{n} \stackrel{w}{\longrightarrow} \lambda^{-1} x$. Since $F$ has weakly sequentially closed graph, we have $y \in F(x)$, which means that $x \in \lambda F(x)$.

Q.E.D.

Our first result is of Leray-Schauder type.

Theorem 3.1 Let $E$ be a Banach space, $\Omega$ be a non-empty, closed, convex subset of $E$. and $U$ be a weakly open subset of $\Omega$ with $\theta \in U$. Assume $\Phi$ a MNWC on $E$ and $F: \overline{U^{w}} \rightarrow P_{\mathrm{cv}}(\Omega)$ has a weakly sequentially closed graph. In addition, suppose $F$ is $\Phi$-condensing and $F\left(\overline{U^{w}}\right)$ is bounded. Then, either

$\left(A_{1}\right) \quad F$ has a fixed point, or

$\left(A_{2}\right)$ there is a point $x \in \partial_{\Omega} U$ (the weak boundary of $U$ in $\Omega$ ) and $\lambda \in(0,1)$ with $x \in \lambda F(x)$.

Remark 3.1 (a) In view of the convexity, the set is weakly closed and therefore the weak closure of $U$ and the weak closure of $U$ in $\Omega$ coincide for any $U \subset \Omega$.

(b) For $U \subset \Omega$, we have $\partial_{\Omega} U=\overline{U^{w}} \cap \overline{\Omega \backslash U^{w}}$.

Proof. Suppose $\left(A_{2}\right)$ does not hold and $F$ does not have a fixed point in $\partial_{\Omega} U$ (otherwise, we are finished, i.e. $A_{1}$ occurs). Let $D$ the set defined by

$$
D=\left\{x \in \overline{U^{w}}: x \in \lambda F(x) \text {, for some } \lambda \in[0,1]\right\}
$$

$D$ is non-empty and bounded, because $\theta \in D$ and $F\left(\overline{U^{w}}\right)$ is bounded. We have

$$
\begin{aligned}
& D \subset \operatorname{conv}(\{\theta\} \cup F(D)) \text {. So, } \Phi(D) \neq 0 \text { implies } \\
& D \subset \operatorname{conv}(\{\theta\} \cup F(D)) \\
&=\left\{x \in \overline{U^{w}}: x \in \lambda F(x), \text { for some } \lambda \in[0,1]\right\}
\end{aligned}
$$

which is a contradiction. Hence, $\Phi(D)=0$ and $D$ is weakly relatively compact. Now, we prove that $D$ is weakly sequentially closed. For that, let $\left(x_{n}\right)_{n}$ a sequence of $D$ such that $x_{n} \stackrel{w}{\longrightarrow} x, x \in \overline{U^{w}}$. For all $n \in \mathbb{N}$, there exists a $\lambda_{n} \in[0,1]$ such that $x_{n}=\lambda_{n} F\left(x_{n}\right)$. $\lambda_{n} \in[0,1]$, we can extract a subsequence $\left(\lambda_{n_{j}}\right)_{j}$ such that $\lambda_{n_{j}} \rightarrow \lambda \in[0,1]$. We put $x_{n_{j}}=\lambda_{n_{j}} y_{n_{j}}$, where $y_{n_{j}} \in F\left(x_{n_{j}}\right)$. Applying Lemma 3.1, we deduce that $\underline{x \in D}$. Let $x \in \overline{U^{w}}$, be weakly adherent to $D$. Since $\overline{D^{w}}$ is weakly compact, by the Eberlein-Šmulian theorem [13, Theorem 8.12.4, p. 549], there exists a sequence $\left(x_{n}\right)_{n} \subset D$ such that $x_{n} \stackrel{w}{\longrightarrow} x$, so $x \in D$. Hence $D^{w}=D$ and $D$ is a weakly closed subset of the weakly compact set $U^{w}$. Therefore $D$ is weakly compact. Because $\bar{E}$ endowed with its weak topology is a Hausdorff locally convex space, we have that $E$ is completely regular $[17$, p. 16]. Since $D \cap(\Omega \backslash U)=\varnothing$, then by [16, p. 146], there is a weakly continuous function $\varphi: \Omega \rightarrow[0,1]$, such that $\varphi(x)=1$ for $x \in D$ and $\varphi(x)=0$ for $x \in \Omega \backslash U$. Since $\Omega$ is convex, $\theta \in \Omega$, and $F$ with nonempty convex values, we can define the multi-valued map $F^{*}: \Omega \rightarrow P_{\mathrm{cv}}(\Omega)$ by:

$$
F^{*}(x)=\left\{\begin{array}{l}
\varphi(x) F(x)+, \text { if } x \in \overline{U^{w}}, \\
\{\theta\}, \text { if } x \in \Omega \backslash \overline{U^{w}}
\end{array}\right.
$$

Clearly, $F^{*}(\Omega)$ is bounded. Because $\partial_{\Omega} U=\partial_{\Omega} \overline{U^{w}}$, $[0,1]$ is compact, $\varphi$ is weakly continuous and $F$ has a weakly sequentially closed graph, by Lemma 3.1, we have $F^{*}$ has weakly sequentially closed graph. Let $X \subset \Omega$, bounded. Then, since

$$
F^{*}(X) \subset \operatorname{conv}\left(\{\theta\} \cup F\left(X \cap \overline{U^{w}}\right)\right)
$$

we have

$$
\Phi\left(F^{*}(X)\right) \leq \Phi\left(\left(X \cap \overline{U^{w}}\right)\right) \leq \Phi(F(X))
$$

and $\Phi\left(F^{*}(X)\right)<\Phi(X)$ if $\Phi(X) \neq 0$. So, $F^{*}$ is $\Phi$-condensing. Therefore, all of the assumptions of Theorem 2.3 are satisfied for $F^{*}$. Consequently there exists $x_{0} \in \Omega$ with $x_{0} \in F^{*}\left(x_{0}\right)$. If $x_{0} \notin U, \Phi\left(x_{0}\right)=0$ and $x_{0}=\theta$, which contradicts the hypothesis $\theta \in U$. Then $x_{0} \in U$ and $x_{0} \in \Phi\left(x_{0}\right) F\left(x_{0}\right)$, which implies that $x_{0} \in D$, and so $\varphi\left(x_{0}\right)=1$ and the proof is complete.

Q.E.D.

Remark 3.2 (a) Theorem 3.1 extends Theorem 2.5 in [4] and shows that the condition $F$ has weakly closed graph can be replaced by $F$ has weakly sequentially closed graph.

(b) Theorem 3.1 extends and improves Theorem 2.4 in [4] and shows that the condition $\overline{U^{w}}$ is weakly compact in the statement of this theorem is redundant.

(c) Theorem 3.1 extends Theorem 3.3 in [8] in the context of single-valued and weakly sequentially continuous maps to the case of multi-valued maps with weakly sequentially closed graph.

Corollary 3.1 Let $E$ be a Banach space, $\Omega$ be a non-empty, closed, convex subset of $E$. and $U$ be a weakly open subset of $\Omega$ with $\theta \in U$. Assume $\Phi$ a MNWC on $E$ and $F: \overline{U^{w}} \rightarrow P_{\mathrm{cv}}(\Omega)$ has a weakly sequentially closed graph, $\Phi$-condensing with $F\left(\overline{U^{w}}\right)$ is bounded. In addition, suppose F satisfying the LeraySchauder boundary condition

$$
x \notin \lambda F(x) \text { for every } x \in \partial_{\Omega} U \text { and } \lambda \in(0,1)
$$


then $F$ has a fixed point in $\overline{U^{w}}$.

Corollary 3.2 Let $E$ be a Banach space, $\Omega$ be a non-empty, closed, convex subset of $E$. and $U$ be a weakly open subset of $\Omega$ with $\theta \in U$. Assume that $F: \overline{U^{w}} \rightarrow P_{\mathrm{cv}}(\Omega)$ a weakly completely continuous map with $F\left(\overline{U^{w}}\right)$ is bounded. In addition, suppose $F$ satisfying the Leray-Schauder boundary condition

$x \notin \lambda F(x)$ for every $x \in \partial_{\Omega} U$ and $\lambda \in(0,1)$

then $F$ has a fixed point in $\overline{U^{w}}$.

Proof. Since $F$ is weakly completely continuous, it follows that $F$ is $\Phi$-condensing on $\Omega$ for any MNWC on $E$. Now, it suffices to apply the Theorem 3.1. Q.E.D. We now use Theorem 2.4 to obtain a nonlinear alternative of Leray-Schauder type for multi-valued 1-set weakly contractive maps.

Theorem 3.2 Let $\Omega$ be a non-empty, closed, convex subset of a Banach space $E$, let $U$ be a weakly open subset of $\Omega$ with $\theta \in U$. Assume $\Phi$ a positive homogenous MNWC on $E, \quad F: \overline{U^{w}} \rightarrow P_{\mathrm{cv}}(\Omega)$ has a weakly sequentially closed graph, $\Phi$-nonexpansive, $F\left(\overline{U^{w}}\right)$ is bounded and (1) holds on $\overline{U^{w}}$. In addition, suppose $F$ satisfies the following Leray-Schauder condition

$$
x \notin \lambda F(x) \quad \text { for every } x \in \partial_{\Omega} U \text { and } \lambda \in(0,1)
$$

then $F$ has a fixed point in $\overline{U^{w}}$.

Proof. Let $F_{n}=t_{n} F$, for $n=1,2, \cdots$, where $\left(t_{n}\right)_{n}$ is a sequence of $(0,1)$ such that $t_{n} \rightarrow 1$. Since $\theta \in \Omega$ and $\Omega$ is convex, it follows that $F_{n}: \overline{U^{w}} \rightarrow P_{\mathrm{cv}}(\Omega)$. Also $F_{n}$ is $\Phi$-condensing and has a weakly sequentially closed graph. Suppose that $y_{n} \in \lambda_{n} F_{n}\left(y_{n}\right)$ for some $y_{n} \in \partial_{\Omega} U$ and for some $\lambda_{n} \in(0,1)$. Then we have $y_{n} \in \lambda_{n} t_{n} F_{n}\left(y_{n}\right)$ which contradicts the hypothesis (2) since $\lambda_{n} t_{n} \in(0,1)$. Now, applying Corollary 3.1, the remainder of the proof is similar to that of Theorem 2.4. Q.E.D.

Remark 3.3 Theorem 3.2 extends and improves Theorem 3.4 in [8].

In applications, it is extremely difficult to construct a weakly open set $U$ as in Theorem 3.1, so we are motivated to construct a Furi-Pera type fixed point theorems ([18]) for a multi-valued mapping $F: M \rightarrow 2^{E}$ with weakly sequentially closed graph. Here $M$ is a closed convex subset of $E$ with (possible) an empty weak interior. The last mentioned case is very important in applications, mainly for the investigation of boundary value problems on noncompact intervals (see [18]).

Our next result extends and improves Theorem 2.4 in
[6].

Theorem 3.3 Let $E$ be a separable Banach space, $\Omega$ a closed convex subset of $E$, and $M$ a closed convex subset of $\Omega$ with $\theta \in M$. Also, assume

$F: M \rightarrow P_{\mathrm{cv}}(\Omega)$ has weakly sequentially closed graph and weakly compact map. In addition, assume the following conditions are satisfied:

There exists a weakly sequentially continuous retraction $r: E \rightarrow M$

There exists $\delta>0$ and a weakly compact set $M_{\delta}$ with

$K_{\delta}=\{x \in E: d(x, M) \leq \delta\} \subseteq M_{\delta}$ here $d(x, y)=\|x-y\|$

for any $K_{\varepsilon}=\{x \in E: d(x, M) \leq \varepsilon, 0<\varepsilon \leq \delta\}$, if

$\left\{\left(x_{j}, \lambda_{j}\right)\right\}_{j=1}^{\infty}$ is a sequence in $M \times[0,1]$ with

$x_{j} \stackrel{w}{\longrightarrow} x \in \partial_{K_{\varepsilon}} M, \lambda_{j} \rightarrow \lambda$ and $x \in \lambda F(x) 0 \leq \lambda<1$

then, $\lambda_{j} F\left(x_{j}\right) \subseteq M$ for $j$ sufficiently large here $\partial_{K_{\varepsilon}} M$ denotes the weak boundary of $M$ in $K_{\varepsilon}$.

Then $F$ has a fixed point in $M$.

Proof. Consider

$$
N=\{x \in \Omega, x \in \operatorname{Fr}(x)\}
$$

We first show $N \neq \varnothing$. Notice $\operatorname{Fr}: \Omega \rightarrow P_{\mathrm{cv}}(\Omega)$ is weakly compact. Since $r$ is weakly sequentially continuous, $M$ is weakly closed, and $F$ has weakly sequentially closed graph, it follows that $\mathrm{Fr}$ has weakly sequentially closed graph. Theorem 2.2 implies that $\mathrm{Fr}$ has a fixed point, so $N \neq \varnothing$ Next we show that $N$ is weakly compact. Indeed, $N \subseteq \operatorname{Fr}(\Omega) \subseteq F(M)$, so $N$ is relatively weakly compact. Now, let $\left(x_{n}\right)_{n}$ a sequence of $N$ such that $x_{n} \stackrel{w}{\longrightarrow} x, x \in E$. For all $n \in \mathbb{N}$ we have $x_{n} \in \operatorname{Fr}\left(x_{n}\right)$ and $r\left(x_{n}\right) \stackrel{w}{\longrightarrow} r(x)$ in $M$. Because $F$ has weakly sequentially closed graph, $x \in \operatorname{Fr}(x)$. Hence, $x \in N$ and $N$ is weakly sequentially closed. Applying again the Eberlein-Šmulian theorem [13, theorem 8.12.4, p. 549], we obtain that $N$ is weakly compact. We now show $M \cap N=\varnothing$. To do this, we argue by contradiction and we use some ideas in [18]. Suppose $M \cap N=\varnothing$. Then since $N$ is compact and $M$ is closed we have by [19, p. 65] that $d(N, M)=\inf \{\|x-y\|: x \in N, y \in M\}>0$. Thus, there exists $\varepsilon, 0<\varepsilon \leq \delta$, with $K_{\varepsilon} \cap N=\varnothing$, here $K_{\varepsilon}=\{x \in E, d(x, M) \leq \varepsilon\}$. We have $K_{\varepsilon}$ is closed, convex, so weakly closed and $K_{\varepsilon} \subseteq M_{\delta}$. Using (4), we obtain $K_{\varepsilon}$ is weakly compact. Because $E$ is separable, the weak topology on $K_{\varepsilon}$ is metrizable (see [20]), and 
let $\rho$ denote this metric. For $i \in\{1,2, \cdots\}$, let

$$
U_{i}=\left\{x \in K_{\varepsilon}, \rho(x, M)<\varepsilon / i\right\}
$$

We fix $i \in\{1,2, \cdots\}$. Now $U_{i}$ is open in $K_{\varepsilon}$ with respect to the topology generated by $\rho$, and so $U_{i}$ is weakly open in $K_{\varepsilon}$. Also we have

$$
\overline{U_{i}^{w}}=\overline{U_{i}^{\rho}}=\left\{x \in K_{\varepsilon}, \rho(x, M) \leq \varepsilon / i\right\}
$$

and

$$
\partial_{K_{\varepsilon}} U_{i}=\left\{x \in K_{\varepsilon}, \rho(x, M)=\varepsilon / i\right\}
$$

Since $d(N, M)>\varepsilon$, then $N \cap \overline{U_{i}^{w}}=\varnothing$. Applying Corollary 3.2, we get that there exists $\lambda \in(0,1)$ and $y_{i} \in \partial_{K_{\varepsilon}} U_{i}$ such that $y_{i} \in \lambda_{i} \operatorname{Fr}\left(y_{i}\right)$. In particular, since $y_{i} \in \partial_{K_{\varepsilon}} U_{i}$, then

$$
\lambda_{i} \operatorname{Fr}\left(y_{i}\right) \not \subset M \text { for each } i \in\{1,2, \cdots\}
$$

Now we investigate

$$
R=\{x \in E: x \in \lambda F r(x) \text {, for some } \lambda \in[0,1]\}
$$

$R$ is non-empty, because $\theta \in R$. Also,

$R \subseteq \overline{\operatorname{Conv}}(F(M) \cup\{\theta\})$, so by the Krein-Šmulian theorem (see [14, p. 434]), $R$ is relatively weakly compact. Since $F r$ has weakly sequentially closed graph and $[0,1]$ is compact, we deduce by Lemma 3.1 that $R$ is weakly sequentially closed. This together with

$$
\rho\left(y_{j}, M\right)=\frac{\varepsilon}{j}, \lambda_{j} \in[0,1] \text { for } j \in\{1,2, \cdots\}
$$

implies that we may assume without loss of generality that

$$
\lambda_{j} \rightarrow \lambda_{0} \text { and } y_{j} \stackrel{w}{\rightarrow} y_{0} \in \overline{M^{w}} \cap \overline{K_{\varepsilon} \backslash M^{w}}=\partial_{K_{\varepsilon}} M
$$

Also since $y_{j} \in \lambda_{j} \operatorname{Fr}\left(y_{i}\right)$ we have $y_{0} \in \lambda_{0} \operatorname{Fr}\left(y_{0}\right)$. If $\lambda_{0}=1$, then $y_{0} \in \operatorname{Fr}\left(y_{0}\right)$, which contradicts

$M \cap N=\varnothing$. Thus, $\lambda_{0} \in[0,1)$. But (5) with

$x_{j}=r\left(y_{i}\right) \in M, x=y_{0}=r\left(y_{0}\right) \in \partial_{K_{\varepsilon}} M$ implies

$\lambda_{j} \operatorname{Fr}\left(y_{i}\right) \subseteq M$ for $j$ sufficiently large. This contradicts (6). Thus $M \cap N=\varnothing$. Accordingly, there exists $x \in M$ such that $x \in \operatorname{Fr}(x)=F(x)$

Q.E.D.

Remark 3.4 (a) If $E^{*}$ is separable and $M$ is weakly compact, then there exists a weakly continuous and so weakly sequentially continuous retraction onto $M$ (see [21]).

(b) If $E$ is reflexive then it suffices to take $F(M)$ is bounded, since a subset of a reflexive Banach space is weakly compact iff it is closed in the weak topology and bounded in the norm topology.

Remark 3.5 (a) Theorem 3.3 extends Theorem 2.5 in [7].

(b) As a corollary of Theorem 3.3 we find Theorem 2.6 in [8].

Remark 3.6 If in the statements of Theorem 3.3, the convex set $\Omega$ is weakly compact, then a special case of (5) which is useful in applications is

If $\left\{\left(x_{j}, \lambda_{j}\right)\right\}_{j=1}^{\infty}$ is a sequence in $M \times[0,1]$ with $x_{j} \stackrel{w}{\longrightarrow} x, \lambda_{j} \rightarrow \lambda$ and $x \in \lambda F(x)$ with $0 \leq \lambda<1$, then $\lambda_{j} F\left(x_{j}\right) \subseteq M$ for $j$ sufficiently large..

Remark 3.7 The set $M$ can be with void weak interior.

\section{References}

[1] G. Bonanno and S. A. Marano, "Positive Solutions of Elliptic Equations with Discontinuous Nonlinearities", Topological Methods in Nonlinear Analysis, Vol. 8, 1996, pp. 263-273.

[2] D. Averna and S. A. Marano, "Existence of Solutions for Operator Inclusions: a Unified Approach,” Rendiconti del Seminario Matematico della Universit di Padova, Vol. 102, 1999, pp. 285-303.

[3] M. Palmucci and F. Papalini, "Periodic and Boundary Value Problems for Second Order Differential Inclusions," Journal of Applied Mathematics and Stochastic Analysis, Vol. 14, No. 2, 2001, pp. 161-182. doi:10.1155/S1048953301000120

[4] D. O'Regan, "Fixed Point Theorems for Weakly Sequentially Closed Maps,” Archivum Mathematicum (Brno) Tomus, Vol. 36, 2000, pp. 61-70.

[5] O. Arino, S. Gautier and J. P. Penot, "A Fixed Point Theorem for Sequentially Continuous Mapping with Application to Ordinary Differential Equations,” Functional Ekvac, Vol. 27, No. 3, 1984, pp. 273-279.

[6] D. O'Regan, "Fixed-Point Theory for Weakly Sequentially Continuous Mapping,” Mathematical and Computer Modelling, Vol. 27, No. 5, 1998, pp. 1-14.

[7] D. O'Regan, “A Continuation Method for Weakly Condensing Operators," Zeitschrift fr Analysis und ihre Anwendungen, Vol. 15, 1996, pp. 565-578.

[8] A. Ben Amar and M. Mnif, "Leray-Schauder Alternatives for Weakly Sequentially Continuous Mappings and Application to Transport Equation,” Mathematical Methods in The Applied Sciences, Vol. 33, No. 1, 2010, pp. 80-90.

[9] A. Ben Amar, A. Jeribi and M. Mnif, "Some Fixed Point Theorems and Application to Biological Model," $\mathrm{Nu}$ merical Functional Analysis and Optimization, Vol. 29, No. 1, 2008, pp. 1-23. doi:10.1080/01630560701749482.

[10] M. A. Taoudi, “Krasnosel'skii Type Fixed Point Theorems under Weak Topology Features,” Nonlinear Analysis, Vol. 72, No. 1, 2010, pp. 478-482. 
doi:10.1016/j.na.2009.06.086.

[11] F. S. DeBlasi, "On a Property of the Unit Sphere in Banach Space,” Bulletin Mathématiques de la Société des Sciences Mathématiques de Roumanie, Vol. 21, 1977, pp. 259-262.

[12] J. Himmelberg, "Fixed Points of Multifunctions,” Journal of Mathematical Analysis and Applications, Vol. 38, No. 1, 1972, pp. 205-207. doi:10.1016/0022-247X(72)90128-X

[13] R. E. Edwards, "Functional Analysis, Theory and Applications,” Reinhart and Winston, New York, 1965.

[14] N. Dunford and J. T. Schwartz, "Linear Operators: Part I,” Intersciences, New York, 1958.

[15] L. Gorniewicz, "Topological Fixed Point Theory of Multivalued Mappings,” 2nd Edition, Springer, New York, 2006.
[16] I. M. James, “Topological and Uniform Spaces,” Springer-Verlag, New York, 1987.

[17] H. H. Schaefer, “Topological Vector Spaces,” Macmillan Company, New York, 1966

[18] M. Furi and P. Pera, “A Continuation Method on Locally Convex Spaces and Applications to Ordinary Differential Equations on Noncompact Intervals," Annales Polonici Math-Ematici, Vol. 47, 1987, pp. 331-346.

[19] K. Floret, “Weakly Compact Sets," Lecture Notes in Mathematics.

[20] E. Zeidler, "Nonlinear Functional Analysis and Its Applications,” Vol. I, Springer, New York, 1986.

[21] G. J. O. Jameson, “An Elementary Proof of the Arens and Borusk Extension Theorems," Journal of the London Mathematical Society, Vol. 14, No. 2, 1976, pp. 364-368. doi:10.1112/jlms/s2-14.2.364 